\title{
Improving stability of zinc nanoparticles in chitosan solution with a nanosecond pulsed laser
}

\begin{abstract}
A green method is presented to fabricate zinc nanoparticles using laser ablation synthesis in a natural polymer solution. The main aims are to reduce the zinc nanoparticles size and to increase their stability by using different ablation times in a chitosan solution as a natural polymer stabilizer. A Nd:YAG pulsed laser $(532 \mathrm{~nm})$ of energy $60 \mathrm{~mJ} /$ pulse, with a pulse duration of $10 \mathrm{~ns}$ was used to ablate a zinc plate immersed in chitosan solution from which the nanoparticles were produced. The average particle sizes as well as the volume fraction of the zinc nanoparticles in the solution were measured at 5, 10, 15, $30 \mathrm{~min}$ of ablation time. The results showed that the particle size decreased from 9.43 to $5.04 \mathrm{~nm}$ with spherical morphology and its volume fraction increased from $0.24 \times 10-7$ to $8.5 \times 10-7$ as the ablation time progressed from 5 to $30 \mathrm{~min}$ respectively. As a comparative study, a reasonable decrease was observed in the particle size for chitosan than distilled water. It was also noted that the stability and dispersion of zinc nanoparticles were much higher in the chitosan solution compared to water. In addition, the COSMO (conductor-like screening model) solvation model was used to compare the enthalpies of $\mathrm{Zn}-\mathrm{NPs}$ in different media. Obtained results confirmed their stability was higher when merged in a natural polymer compared to water.
\end{abstract}

Keyword: Zinc nanoparticles; Chitosan solution; Nanosecond pulsed laser 